\title{
BMJ Open Comparative effectiveness of inhaled corticosteroids for paediatric asthma: protocol for a systematic review and Bayesian network meta-analysis
}

\author{
Masato Takeuchi, ${ }^{1}$ Hirotsugu Kano, ${ }^{2}$ Kenzo Takahashi, ${ }^{3}$ Tsutomu Iwata ${ }^{4}$
}

To cite: Takeuchi M, Kano $\mathrm{H}$, Takahashi K, et al. Comparative effectiveness of inhaled corticosteroids for paediatric asthma: protocol for a systematic review and Bayesian network metaanalysis. BMJ Open 2015;5: e008501. doi:10.1136/ bmjopen-2015-008501

- Prepublication history and additional material available. To view please visit the journal (http://dx.doi.org/10. 1136/bmjopen-2015008501).

Received 16 April 2015 Revised 9 September 2015 Accepted 5 October 2015

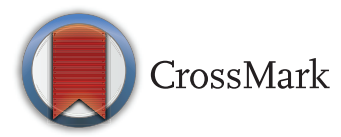

${ }^{1}$ Department of Pediatrics, Kikkoman General Hospital, Noda, Chiba, Japan ${ }^{2}$ Department of Pediatrics, Teikyo University School of Medicine University HospitalMizonokuchi, Kawasaki, Kanagawa, Japan

${ }^{3}$ Graduate School of Public Health, Teikyo University, Itabashi, Tokyo, Japan ${ }^{4}$ Faculty of Child Studies, Department of Education for Childcare, Tokyo Kasei University, Sayama, Saitama, Japan

Correspondence to Dr Masato Takeuchi; masatotakeuchi@gmail.com

\section{ABSTRACT}

Introduction: Use of inhaled corticosteroid (ICS) is the mainstream maintenance therapy for paediatric asthma. Several forms of ICS are available, but the relative effectiveness among ICS has not been well investigated in published, randomised, controlled trials. The paucity of direct comparisons between ICS may have resulted in insufficient estimation in former systematic reviews/meta-analyses. To supplement the information on the comparative effectiveness of ICS for paediatric asthma, we plan to conduct a network metaanalysis that will enable summary of direct and indirect evidence.

Methods and analysis: We will retrieve randomised, controlled trials that examined the effectiveness of ICS for paediatric asthma from the PubMed and Cochrane Central Register of Controlled Trials. After one author scans the title and abstract for eligible studies, two authors will independently review study data and assess the quality of the study. Studies of children ( $\leq 18$ years old) with chronic asthma or recurrent wheezing episodes will be included if they used ICS for $\geq 4$ weeks. We will define a priori core outcomes and supplemental outcomes of paediatric asthma, including exacerbation, healthcare use and pulmonary function.

Studies reporting a minimum of one core outcome will be entered into the systematic review. After the systematic review is performed, extracted data of relevant studies will be synthesised in the Bayesian framework using a random-effects model.

Ethics and dissemination: The results will be disseminated through peer-reviewed publications and conference presentations.

Protocol registration number: UMIN (000016724) and PROSPERO (CRD42015025889).

\section{INTRODUCTION}

Morbidity of paediatric asthma is substantial worldwide. The prevalence of childhood asthma differs among countries, ${ }^{1}$ and up to $20-25 \%$ of children have prescriptions for antiasthma medications in some industrialised countries. ${ }^{2}{ }^{3}$ Data from the USA represent an example of the asthma-related

\section{Strengths and limitations of this study}

- This study will be the first meta-analysis to examine comparative effectiveness of inhaled corticosteroids for paediatric asthma.

- The results of this study will aid clinical decisionmaking for practitioners and will provide the basis of future cost-effective analysis.

- A potential limitation of our study is that it only includes published trials, which may be affected by some bias (eg, publication bias).

burden in children. These data show that, in 1 year, asthma causes exacerbations in $57 \%$ of paediatric patients, 12.8 million missed school days, 198000 hospitalisations (the third cause of all childhood hospitalisations) and 185 deaths. ${ }^{1}$

Asthma is characterised by chronic inflammation of the airways. ${ }^{5}$ Therefore, for control of airway inflammation, regular maintenance therapy is required in most patients. ${ }^{6} 7$ Use of inhaled corticosteroid (ICS) is the mainstream of asthma treatment in adults and children. ICS use achieves asthma control and this therapy leads to fewer exacerbations, emergency department visits and hospitalisations. ${ }^{8}$ ICS also improve other outcome measures of asthma, such as pulmonary function $^{9}$ and quality of life (QOL) of patients. ${ }^{10}$

There are several forms of ICS for paediatric patients. Fluticasone propionate (FP), hydrofluoroalkane-134a beclometasone dipropionate (HFA-BDP), budesonide (BUD) and ciclesonide are commonly prescribed ICS for paediatric asthma. ${ }^{11}$ The relative effectiveness of these agents is estimated by their potency in vitro. ${ }^{12}$ On the basis of the in vitro observations, the effectiveness of different ICS is often assumed to be similar (ie, 1:1 ratio in equivalent dose) to that in vivo. However, these ICS have different properties. ${ }^{8}$ FP has a potent affinity for steroid 
receptors with a long half-life, ${ }^{13}$ HFA-BDP is composed of small particles and can be delivered to small airways ${ }^{14}$ and BUD suspension is easy to use in children who are not cooperative with inhalation therapy ${ }^{15}$ Owing to differences in formulations and delivery systems, the effectiveness of ICS can differ clinically. ${ }^{16} \mathrm{~A}$ medical database study from the USA reported that asthma control might be better in patients with HFA-BDP than in those with FP. ${ }^{17}$ However, few studies have compared different types of ICS directly. ${ }^{18}$ One systematic review concluded that there was little evidence of the comparative effectiveness of ciclesonide with other ICS among adult patients. ${ }^{19}$ That review was restricted to small, phase 2 studies of low power. The authors found only five randomised, controlled trials (RCTs) with a total of 84 patients. ${ }^{19}$

One strategy to improve the statistical power of small studies is to conduct a meta-analysis. ${ }^{20}$ However, as aforementioned, studies comparing different classes of ICS are limited. The majority of clinical trials compared ICS with other classes of drugs (eg, antileukotrienes or ICS/ long-acting $\beta$-agonist (LABA) combinations) or placebo. The paucity of trials of direct comparisons makes it difficult to perform a conventional meta-analysis (hereafter, we use the term 'pairwise meta-analysis'). Recently, a novel meta-analytic technique called a network meta-analysis (NMA) was developed, and this enables results of trials to be combined in a direct as well as an indirect manner. ${ }^{21-23}$

In this context, we plan to conduct an NMA to address the following open question: Are there any differences in effectiveness among ICS for paediatric asthma?

\section{METHODS AND ANALYSIS \\ Goal of the study}

We aim to evaluate the comparative effectiveness of different ICS for paediatric asthma. For this purpose, we will use the NMA approach to synthesise two types of clinical trials together: trials comparing different ICS directly and trials comparing ICS with other classes of intervention (eg, antileukotrienes or placebo).

\section{PRISMA-P 2015/PRISMA Extension statement}

For developing this protocol, we referred to the preferred reporting items for systematic review and meta-analysis protocols (PRISMA-P) 2015 statement, ${ }^{24}$ which is a guide for standard reporting of systematic review protocols. Corresponding to the PRISMA-P statement, we have registered this protocol at PROSPERO ${ }^{25}$ and UMIN. ${ }^{26}$ Although, overall, we adhere to the PRISMA-P statement, the method of dealing with publication bias (item 16) is not specified in this protocol. This is because identification of publication bias is more complex in NMA owing to limited numbers of studies for each pairwise comparison, heterogeneity and other limitations, ${ }^{27}$ and there are no formal techniques to detect or assess the extent of publication bias. NMA is, however, a rapidly evolving research area and, if standard approaches are established at the time of our final report, we will be ready to use those skills.

We also referred to the PRISMA extension statement, which incorporates reporting of NMA, ${ }^{27}$ and this protocol was partially developed with the help of the PRISMA extension statement.

Inclusion criteria: participants, interventions, comparisons and outcomes

Participants

Studies of children ( $\leq 18$ years old) with mild-to-moderate chronic or persistent asthma will be included. We will include studies exclusively comprising of paediatric patients, and those involving adult and paediatric patients if data of paediatric age groups are accessible and can be extracted.

This meta-analysis will also include studies of 'children with recurrent wheeze' or 'preschool wheezers'. Currently, the diagnosis of asthma in young children is challenging because there are no universally accepted diagnostic criteria. ${ }^{28}$ Only a subset of young children with recurrent wheezing episodes later develops physician-diagnosed asthma. ${ }^{29}{ }^{30}$ In addition, there is a wide range of differential diagnoses in recurrent wheeze that mimic paediatric asthma, such as cystic fibrosis, congenital malformation of the airways and foreign body aspiration. ${ }^{31}$ Therefore, children with recurrent wheeze may or may not have asthma. The likelihood of asthma in such patients depends on the presence/absence of risk factors (eg, family history).$^{32}$ Despite these problematic issues, we have decided to enrol children with recurrent wheeze for the following three reasons. First, recurrent wheezing is a major risk factor of asthma. As shown in studies of the Asthma Predictive Index, the combination of wheezing episodes ( $\geq 3$ episodes/year) and other criteria is strongly associated with the risk of asthma (up to $77 \%$ chance of active asthma). ${ }^{30}$ Second, in addition to symptoms and risk factors, the therapeutic response is often important for diagnosis of paediatric asthma, ${ }^{32}{ }^{33}$ and empirical evidence indicates that children with recurrent wheeze may benefit from regular ICS use. ${ }^{31}$ Finally, previous systematic reviews/ meta-analyses have not often distinguished children with asthma from those with recurrent wheeze. ${ }^{34}$ Owing to these reasons, we consider that children with asthma and those with recurrent wheeze share similar (although not identical) clinical characteristics and responses to ICS therapy. We will include only data of physiciandiagnosed wheezing ( $\geq 3$ times, separately) to ensure the consistency of patients' symptoms. In trials of children with recurrent wheeze, we carefully review (1) whether the risk factors of asthma (eg, atopic status or family history) are described, and (2) whether differential diagnoses of wheeze are investigated. If these issues are insufficiently examined or documented, the authors will discuss whether such reports will be eligible for inclusion into the meta-analysis. 


\section{Interventions}

We will include RCTs to examine the effectiveness of ICS in asthmatic children for $\geq 4$ weeks. We will only include studies using ICS without co-interventions because the effectiveness of ICS is difficult to assess separately in trials with co-intervention (eg, ICS/LABA combination therapy). We will limit studies evaluating the effectiveness of ICS in current use (ie, studies of ICS that are no longer used, such as HFA-chlorofluorocarbon, will be excluded). Therefore, this study will include the BDP HFA-metered dose inhaler (MDI), BUD (dry powder inhaler (DPI) and nebules), ciclesonide (HFA-MDI), flunisolide (HFA-MDI), FP (HFA-MDI and DPI) and mometasone furoate (MDI and DPI).

\section{Comparisons}

This study will include clinical trials comparing one ICS with other active or inactive intervention(s), such as other types of ICS, other classes of drugs (eg, antileukotrienes) or placebo. The comparator should also be a single intervention because of the reason aforementioned.

\section{Outcomes}

In meta-analyses, researchers often declare the primary end point of the study. ${ }^{35}$ However, this practice is difficult in asthma studies. ${ }^{36}$ There are several domains in asthma control, such as a pulmonary function test or symptoms (eg, exacerbation), and, according to expert opinion, no single primary end point is recommended for assessment of responses to asthma. ${ }^{37}$ Therefore, our planned study will not define a single primary end point but, instead, it will examine different end points to determine a more complete understanding of asthma control by ICS (table 1). ${ }^{36}$

Study outcomes should be clinically relevant, and, ideally, they should be patient centred. ${ }^{38}$ Additionally, outcomes of a sufficiently large number of trials should be pooled in the analysis. Summarising a large sample size would lead to more precise and confident estimation and, in NMA, combining small sample size studies could result in biased estimates. ${ }^{39}$ From these perspectives, we will not include studies that exclusively examined biomarkers, QOL, or severity scores for the following reasons. First, how these outcomes correlate with the clinical benefit has yet to be established, and the magnitude of benefit of these outcomes is difficult to interpret for patients and even for healthcare professionals. ${ }^{37}$ Second, a previous systematic review identified a few studies that examined these outcomes in paediatric patients. ${ }^{40}$ Finally, for QOL and severity scores, different formulations are available and they are not interchangeable with each other. ${ }^{41}$

\section{Exclusion criteria}

We will exclude the following literature: abstracts only (eg, conference paper), studies that are not on asthma (eg, viral bronchiolitis), studies examining the doseresponse relationship of ICS (because of technical

Table 1 Core and supplemental outcomes relevant to paediatric asthma

\begin{tabular}{|c|c|c|}
\hline & Core outcomes & Supplemental outcomes \\
\hline Exacerbations & $\begin{array}{l}\text { 1. Systemic corticosteroids for asthma } \\
\text { 2. Asthma-specific hospital admissions } \\
\text { 3. Asthma-specific ED visits (separate UC visits } \\
\text { when these can be differentiated) } \\
\text { 4. Asthma-specific ICU admissions/intubations } \\
\text { 5. Death (all cause and asthma related) }\end{array}$ & (None defined for regular maintenance therapy) \\
\hline $\begin{array}{l}\text { Healthcare } \\
\text { utilisation }\end{array}$ & $\begin{array}{l}\text { 1. Asthma-specific hospital admissions } \\
\text { 2. Asthma-specific ED visits } \\
\text { 3. Asthma-specific outpatient visits } \\
\text { 4. Asthma-specific detailed medication use } \\
\text { (name, dose and duration) } \\
\text { 5. Resource use related to the intervention }\end{array}$ & $\begin{array}{l}\text { 1. Categorisation of asthma-specific outpatient } \\
\text { visits: } \\
\text { Primary care: I. scheduled; II. unscheduled } \\
\text { 2. Respiratory healthcare use } \\
\text { 3. Asthma school absences } \\
\text { 4. Asthma work presenteeism and absenteeism } \\
\text { (WPAI instrument) }\end{array}$ \\
\hline $\begin{array}{l}\text { Pulmonary } \\
\text { physiology }\end{array}$ & Spirometry (without bronchodilator) & $\begin{array}{l}\text { 1. PEF monitoring } \\
\text { 2. Airway responsiveness } \\
\text { 3. Lung volumes } \\
\text { 4. Spirometry (prebronchodilator and } \\
\text { postbronchodilator) } \\
\text { 5. Gas exchange: arterial blood gases and pulse } \\
\text { oximetry }\end{array}$ \\
\hline
\end{tabular}

Based on reference. $^{49}$

ED, emergency department; ICU, intensive care unit; PEF, peak expiratory flow; UC, unscheduled; WPAI, work productivity and activity impairment. 
difficulties in incorporating data into the meta-analysis), safety assessment studies of ICS and short term or intermittent use of ICS.

\section{Literature search}

The primary literature search will rely on PubMed and the Cochrane Central Register of Controlled Trials (CENTRAL). We will enrol all RCTs, including those of cross-over or quasirandomised design, that are published in full-text articles in the English language. We will use medical subject headings and text words related to 'child', 'asthma' and 'ICS' for the literature search. ${ }^{40}$ To ensure literature saturation, we will scan the reference lists of included studies or relevant reviews that are identified through the search.

\section{Selection of studies and extraction of data}

One of the authors (MT) will scan the titles and abstracts of all the literature retrieved by the initial search and select eligible articles for review of the full text. Two other authors (HK and KT) will independently review full-text articles to assess eligibility and select citations to be meta-analysed. Studies that reported at least one core outcome will be selected (shown in table 1). The authors will also extract data independently using a prestandardised data abstraction form. Any disagreements will be resolved by discussion among all the authors. The process of literature selection will be published (eg, web-appendix style).

\section{Quality assessment}

We will assess the quality and risk of bias of eligible studies, such as the method of randomisation, treatment allocation concealment, blinding the outcome assessor and dropouts. For this purpose, the Cochrane risk assessment tool will be used. ${ }^{42}{ }^{43}$ We will also rely on the Grading of Recommendations Assessment, Development and Evaluation approach for quality assessment in cumulative estimates.

\section{Statistical methods}

Figure 1a illustrates the scheme of the proposed pairwise meta-analysis. A pairwise meta-analysis can compare head-to-head trials (figure 1a, A vs B and A vs C), but cannot compare indirect arms (figure $1 \mathrm{a}, \mathrm{B}$ vs $\mathrm{C}$ ). In contrast, NMA can compare indirect arms (figure 1a, B vs $\mathrm{C}$ ). On the basis of a 'consistency assumption', the indirect effect B-C represents the difference between effect A-B and effect A-C (in this case, intervention A is referred to as a common comparator). ${ }^{23}{ }^{44}$ Moreover, when there are head-to-head trials between $\mathrm{B}$ and $\mathrm{C}$ (figure 1b), NMA can combine the direct effect B-C and indirect effect B-C (ie, effect A-B-effect A-C) ${ }^{45}$ In this way, NMA combines all available evidence of direct and indirect comparisons. There is an additional strength in NMA. A pairwise meta-analysis can compare only two interventions at a time. ${ }^{23}$ In the situation shown in figure $1 \mathrm{~b}$, comparison of 'A vs $\mathrm{B}$ vs $\mathrm{C}$ ' is not feasible, (a)

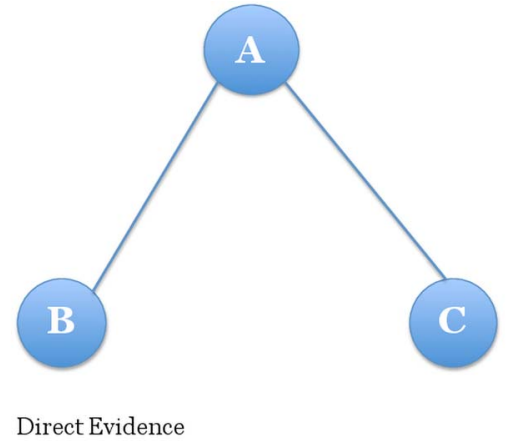

(b)

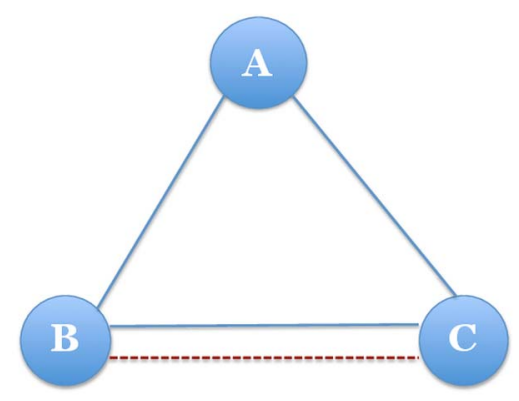
Direct Evidence
Indirect Evidence Combine both Evidence in NMA (if any)

Figure 1 (a) Scheme for pairwise meta-analysis. In this example, the comparison of 'B vs C' is impractical. (b) Scheme for pairwise and network (indirect) meta-analysis. An indirect comparison of ' $B$ vs $C$ ' can be estimated from knowledge of 'A vs $B$ ' and 'A vs $C$ ' trials.

even when direct comparisons exist. In contrast, NMA can compare $\geq 3$ interventions and determine which treatment works best. Further, NMA can compare more complex network loops (figure 2). Figure 2 shows that comparative effectiveness among the ICS' $\mathrm{X}, \mathrm{Y}$ and $\mathrm{Z}$ can be estimated by combining direct evidence (effect B-C) and indirect effects using drug A and placebo as common comparators. On the basis of these strengths in NMA, we will evaluate the comparative effectiveness of ICS by pooling the results from head-to-head trials of ICS and from indirect comparisons among different ICS using placebo or other classes of medications (eg, antileukotriene drugs) as a common comparator.

Statistical analyses will be conducted in a Bayesian hierarchical framework using a random-effects model. ${ }^{46}$ We will use the gemtc package in $\mathrm{R}$ statistical software. ${ }^{478}$ This package uses a method developed by $\mathrm{Lu}$ and Ades. ${ }^{22}$ This package also allows us to check for homogeneity and consistency, which are important assumptions in NMA that combined studies should be similar in clinical and statistical context (often referred to as transitivity assumption ${ }^{23}$ ). The statistical results will be presented in OR (with credible interval) and probability ranking.

If we observe heterogeneity among studies, subgroup analyses will be conducted (see the subsection 'Subgroup analysis' below). As an example of this 


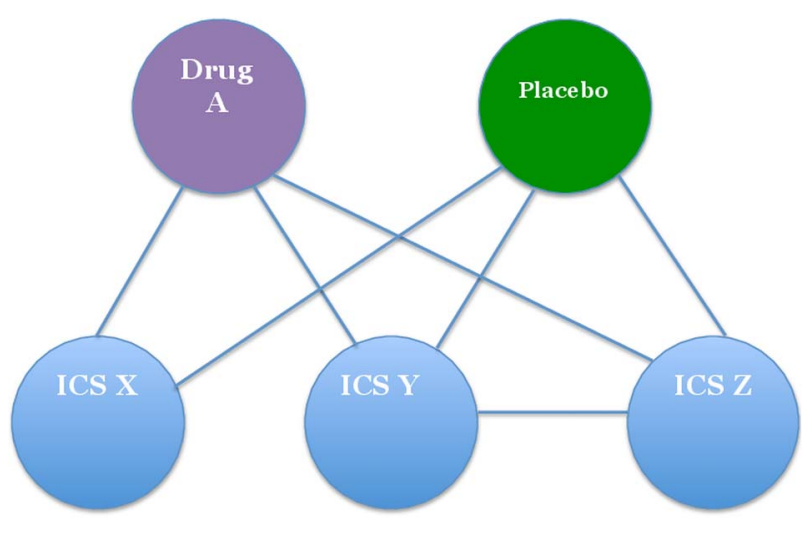

- Direct Evidence

Figure 2 Scheme for a complex network in network meta-analysis (ICS, inhaled corticosteroid).

situation, when the dosage of ICS varies considerably among studies, we will stratify studies of 'low', 'medium' and 'high' dose, ${ }^{18}{ }^{49}$ and combine the results within each strata. The statistical analyses will be performed by one author (MT) on the basis of previous expertise. ${ }^{50}$

The gemtc $\mathrm{R}$ package has a unique function to check local (in) consistency, which we will use for this purpose. We will use $\mathrm{I}^{2}$ statistics to check global (in)consistency. This $\mathrm{R}$ package also prepares a function to generate network geometry, a graphical presentation of the network of evidence, which is an essential item of NMA reporting. ${ }^{27}$

\section{Subgroup analysis}

Heterogeneity is a potential concern in meta-analysis. If heterogeneity is detected, we plan to conduct the following subgroup analyses and will report the results when necessary:

- Patients with chronic asthma versus recurrent wheezers;

- Age groups stratified into three categories $(0-4,5-11$, $\geq 12$ years);

- Children-specific study versus 'children and adult' study;

- Dose stratification into low, medium and high dose.

\section{Role of the funding source}

This study is funded by the Japanese Society of Paediatric Allergy and Clinical Immunology. The funding source has no role in the study design, data collection and analysis, interpretation of results or preparation and submission of the manuscript.

\section{DISCUSSION}

This protocol paper presents the hypothesis, rationale and methodology of our planned study.

The relative potency of different ICS has been the subject of considerable dispute and debate. ${ }^{12}$ Comparative dosing charts among ICS have been proposed, (eg, by an expert panel $)^{49}$ and they rely on comparative efficacy trials in vitro. Few studies have assessed relative therapeutic indices among ICS $^{1851}$ and whether there are clinical differences between ICS remains uncertain. To challenge this open question, we plan to conduct NMA, a newly developed meta-analytic technique.

NMA (also known as a multiple treatment comparison meta-analysis or mixed treatment meta-analysis) has gained popularity in recent years ${ }^{23}{ }^{44}$ in light of comparative effectiveness research (CER). By definition, CER refers to studies that compare the benefits and harms of different interventions. ${ }^{52}$ The objectives of CER include helping physicians use existing treatments and treatment strategies more effectively. ${ }^{53}$ CER also aims to determine which interventions and strategies are most effective, safest, or least costly when multiple options are available. ${ }^{53}$ CER is an emerging study area that is crucial for helping clinical decision-making. However, within the current framework of medicine, limited data are available among different interventions. Comparative efficacy data are often lacking at preapproval and postapproval of medications. ${ }^{53-55}$ To bridge the gap between the needs and the lack of CER studies, new clinical trials or systematic reviews/ meta-analyses, specifically NMA, are the priorities for future research. ${ }^{56}$ Our planned study to determine the comparative effectiveness of ICS for paediatric asthma is in line with the current effort for CER.

Relevance and credibility are two essential components in NMA. ${ }^{57}$ The expected results of our study will be relevant in that they will be applicable to clinical settings of interest to asthmatic patients or healthcare providers. We hope that the results of this NMA study will be credible, providing valid answers to the research question of 'Are there any differences in effectiveness among ICS for paediatric asthma?'.

\section{ETHICS AND DISSEMINATION}

No ethical approval is required because this study will include published clinical trials with no personal data of patients.

The results of this study will be submitted to a peerreviewed journal for publication and will also be presented at future conferences.

Contributors All the authors made substantial contributions to the conception and design of the study. MT prepared the first draft of the article. HK, KT and TI critically revised the manuscript for important intellectual content. All the authors provided final approval of the version to be published.

Funding This study is funded by the Japanese Society of Paediatric Allergy and Clinical Immunology.

Competing interests None declared.

Patient consent Obtained.

Provenance and peer review Not commissioned; externally peer reviewed.

Data sharing statement Additional data is available by emailing the corresponding author. 
Open Access This is an Open Access article distributed in accordance with the Creative Commons Attribution Non Commercial (CC BY-NC 4.0) license, which permits others to distribute, remix, adapt, build upon this work noncommercially, and license their derivative works on different terms, provided the original work is properly cited and the use is non-commercial. See: http:// creativecommons.org/licenses/by-nc/4.0/

\section{REFERENCES}

1. Guilbert T, Moss HM, Lemanske RF Jr. Approach to infants and children with asthma. In; Adkinson NF Jr, Bochner BS, Busse WW, et al. eds. Middleton's allergy: principles and practice. Philadelphia; Saunders, 2009:1319-44.

2. Bianchi M, Clavenna A, Labate L, et al. Anti-asthmatic drug prescriptions to an Italian paedriatic population. Pediatr Allergy Immunol 2009;20:585-91.

3. Bianchi M, Clavenna A, Bonati M. Inter-country variations in anti-asthmatic drug prescriptions for children. Systematic review of studies published during the 2000-2009 period. Eur J Clin Pharmacol 2010;66:929-36.

4. Centers for Disease Control and Prevention. CDC vital signs: Asthma in the US 2011. http://www.cdc.gov/vitalsigns/pdf/201105-vitalsigns.pdf (accessed 16 Mar 2015).

5. Hanania NA. Targeting airway inflammation in asthma: current and future therapies. Chest 2008;133:989-98.

6. Rank MA, Hagan JB, Park MA, et al. The risk of asthma exacerbation after stopping low-dose inhaled corticosteroids: a systematic review and meta-analysis of randomized controlled trials. J Allergy Clin Immunol 2013;131:724-9.

7. Morton RW, Everard ML, Elphick HE. Adherence in childhood asthma: the elephant in the room. Arch Dis Child 2014;99: 949-53.

8. Cerasoli F Jr. Developing the ideal inhaled corticosteroid. Chest 2006;130(1 Suppl):54S-64S.

9. Raissy $\mathrm{HH}$, Kelly HW, Harkins $\mathrm{M}$, et al. Inhaled corticosteroids in lung diseases. Am J Respir Crit Care Med 2013;187:798-803.

10. Murphy KR, Fitzpatrick S, Cruz-Rivera M, et al. Effects of budesonide inhalation suspension compared with cromolyn sodium nebulizer solution on health status and caregiver quality of life in childhood asthma. Pediatrics 2003;112:e212-19.

11. The Thoracic Society of Australia and New Zealand. The Role of Corticosteroids in the Management of Childhood Asthma. 2010. http://www.thoracic.org.au/imagesDB/wysiwyg/Steroidsinasthma 2010.pdf (accessed 16 Mar 2015)

12. Barnes NC. The properties of inhaled corticosteroids: similarities and differences. Prim Care Respir J 2007;16:149-54.

13. Derendorf H, Hochhaus G, Meibohm B, et al. Pharmacokinetics and pharmacodynamics of inhaled corticosteroids. J Allergy Clin Immunol 1998;101:S440-6.

14. Vanden Burgt JA, Busse WW, Martin RJ, et al. Efficacy and safety overview of a new inhaled corticosteroid, QVAR (hydrofluoroalkane-beclomethasone extrafine inhalation aerosol), in asthma. J Allergy Clin Immunol 2000;106:1209-26.

15. Szefler SJ, Eigen H. Budesonide inhalation suspension: a nebulized corticosteroid for persistent asthma. J Allergy Clin Immunol 2002;109:730-42.

16. Baptist AP, Reddy RC. Inhaled corticosteroids for asthma: are they all the same? J Clin Pharm Ther 2009;34:1-12.

17. Colice G, Martin RJ, Israel E, et al. Asthma outcomes and costs of therapy with extrafine beclomethasone and fluticasone. J Allergy Clin Immunol 2013;132:45-54.

18. Kelly HW. Comparison of inhaled corticosteroids: an update. Ann Pharmacother 2009;43:519-27.

19. Dyer MJ, Halpin DM, Stein K. Inhaled ciclesonide versus inhaled budesonide or inhaled beclomethasone or inhaled fluticasone fo chronic asthma in adults: a systematic review. BMC Fam Pract 2006;7:34.

20. Thacker SB. Meta-analysis. A quantitative approach to research integration. JAMA 1988;259:1685-9.

21. Lumley T. Network meta-analysis for indirect treatment comparisons. Stat Med 2002;21:2313-24.

22. Lu G, Ades AE. Combination of direct and indirect evidence in mixed treatment comparisons. Stat Med 2004;23:3105-24.

23. Cipriani A, Higgins JP, Geddes JR, et al. Conceptual and technical challenges in network meta-analysis. Ann Intern Med 2013;159:130-7.

24. Moher D, Shamseer L, Clarke M, et al. Preferred reporting items for systematic review and meta-analysis protocols (PRISMA-P) 2015 statement. Syst Rev 2015;4:1.
25. University of York, Centre for Reviews and Dissemination. http:// www.crd.york.ac.uk/PROSPERO/ (accessed 16 Mar 2015).

26. University hospital Medical Information Network (UMIN) Center http://www.umin.ac.jp/ (in Japanese) (accessed 16 Mar 2015).

27. Hutton B, Salanti G, Caldwell DM, et al. The PRISMA extension statement for reporting of systematic reviews incorporating network meta-analyses of health care interventions: checklist and explanations. Ann Intern Med 2015;162:777-84.

28. Cave AJ, Atkinson LL. Asthma in preschool children: a review of the diagnostic challenges. J Am Board Fam Med 2014;27:538-48.

29. Pescatore AM, Dogaru CM, Duembgen L, et al. A simple asthma prediction tool for preschool children with wheeze or cough J Allergy Clin Immunol 2014;133:111-8.e13.

30. Castro-Rodriguez JA. The Asthma Predictive Index: a very useful tool for predicting asthma in young children. J Allergy Clin Immunol 2010;126:212-16.

31. Ducharme FM, Tse SM, Chauhan B. Diagnosis, management, and prognosis of preschool wheeze. Lancet 2014;383:1593-6.

32. Bacharier LB, Guilbert TW. Diagnosis and management of early asthma in preschool-aged children. J Allergy Clin Immunol 2012;130:287-96.

33. Bacharier LB, Boner A, Carlsen $\mathrm{KH}$, et al. Diagnosis and treatment of asthma in childhood: a PRACTALL consensus report. Allergy 2008;63:5-34

34. Castro-Rodriguez JA, Rodrigo GJ. Efficacy of inhaled corticosteroids in infants and preschoolers with recurrent wheezing and asthma: a systematic review with meta-analysis. Pediatrics 2009;123: e519-25.

35. Rangwalla SC, Waljee AK, Higgins PD. Voting With their Feet (VWF) endpoint: a meta-analysis of an alternative endpoint in clinica trials, using 5-ASA induction studies in ulcerative colitis. Inflamm Bowel Dis 2009;15:422-8.

36. Busse WW, Morgan WJ, Taggart V, et al. Asthma outcomes workshop: overview. J Allergy Clin Immunol 2012;129(3 Suppl): S1-8.

37. Reddel HK, Taylor DR, Bateman ED, et al. An official American Thoracic Society/European Respiratory Society statement: asthma control and exacerbations: standardizing endpoints for clinical asthma trials and clinical practice. Am J Respir Crit Care Med 2009;180:59-99.

38. Frank L, Basch E, Selby JV. The PCORI perspective on patient-centered outcomes research. JAMA 2014;312:1513-14.

39. Odom D, Chirila C, Sherrill B, et al. Practical issues when conducting network meta-analyses with a limited number of studies [abstract]. Montreal, QC, Canada: ISPOR 19th Annual International Meeting, 2014

40. Sinha IP, Williamson PR, Smyth RL. Outcomes in clinical trials of inhaled corticosteroids for children with asthma are narrowly focussed on short term disease activity. PLOS ONE 2009;4 e6276.

41. Parulekar AD, Alobaidy A, Hanania NA. Asthma outcomes revisited. Curr Opin Pulm Med 2013;19:6-12.

42. The Cochrane Collaboration's tool for assessing risk of bias. http:// ohg.cochrane.org/sites/ohg.cochrane.org/files/uploads/Risk\%20of\% 20bias\%20assessment\%20tool.pdf (accessed 16 Mar 2015).

43. Higgins JP, Altman DG, Gotzsche PC, et al. The Cochrane Collaboration's tool for assessing risk of bias in randomised trials. BMJ 2011;343:d5928.

44. Mills EJ, Thorlund K, loannidis JP. Demystifying trial networks and network meta-analysis. BMJ 2013;346:f2914.

45. Caldwell DM, Ades AE, Higgins JP. Simultaneous comparison of multiple treatments: combining direct and indirect evidence. BMJ 2005;331:897-900

46. Dias S, Sutton AJ, Ades AE, et al. Evidence synthesis for decision making 2: a generalized linear modeling framework for pairwise and network meta-analysis of randomized controlled trials. Med Decis Making 2013;33:607-17.

47. GeMTC R package. http://drugis.org/software/r-packages/gemtc (accessed 16 Mar 2015).

48. Neupane B, Richer D, Bonner AJ, et al. Network meta-analysis using R: a review of currently available automated packages. PLoS ONE 2014:9:e115065.

49. National Asthma Education and Prevention Program. Expert Panel Report 3 (EPR-3): Guidelines for the Diagnosis and Management of Asthma-Summary Report 2007. J Allergy Clin Immunol 2007;120(5 Suppl):S94-138.

50. Takeuchi M. Bayesian network meta-analysis suggests a similar effectiveness between a monovalent and a pentavalent rotavirus vaccine: a preliminary report of re-analyses of data from a Cochrane Database Systematic Review. Hum Vaccin Immunother 2014;10:1421-4. 
51. Main C, Shepherd J, Anderson R, et al. Systematic review and economic analysis of the comparative effectiveness of different inhaled corticosteroids and their usage with long-acting beta2 agonists for the treatment of chronic asthma in children under the age of 12 years. Health Technol Assess 2008;12:1-174, iii-iv.

52. Sox HC. Defining comparative effectiveness research: the importance of getting it right. Med Care 2010;48(Suppl):S7-8.

53. Hochman M, McCormick D. Characteristics of published comparative effectiveness studies of medications. JAMA 2010;303:951-8.

54. Goldberg NH, Schneeweiss S, Kowal MK, et al. Availability of comparative efficacy data at the time of drug approval in the United States. JAMA 2011;305:1786-9.
55. Thomas RH, Freeman MK, Hughes PJ. Preapproval and postapproval availability of published comparative efficacy research on biological agents. Am J Health Syst Pharm 2013;70:1250-5.

56. $\mathrm{Li} \mathrm{T}$, Vedula SS, Scherer R, et al. What comparative effectiveness research is needed? A framework for using guidelines and systematic reviews to identify evidence gaps and research priorities. Ann Intern Med 2012;156:367-77.

57. Jansen JP, Trikalinos T, Cappelleri JC, et al. Indirect treatment comparison/network meta-analysis study questionnaire to assess relevance and credibility to inform health care decision making: an ISPOR-AMCP-NPC Good Practice Task Force report. Value Health 2014:17:157-73. 